\title{
Ploidal Inheritance in Dictyostelium discoideum: Stable Haploid, Stable Diploid and Metastable Strains
}

\author{
By M. SUSSMAN AND RAQUEL R. SUSSMAN \\ Brandeis University, Waltham, Massachusetts, U.S.A.
}

(Received 24, August 1961)

\begin{abstract}
SUMMARY
The existence of haploid and diploid Dictyostelium discoideum amoebae has been confirmed. Strains were isolated which displayed widely different ploidal compositions. These appear to fall into three general classes : stable haploid, stable diploid and metastable strains. Their ploidal compositions are clonally inherited. Procedures are described by which strains of differing ploidal composition can be isolated, one from the other. The ploidal composition of a clone is correlated with differences in spore and amoeboid size distributions as well as other morphological and morphogenetic properties.
\end{abstract}

\section{INTRODUCTION}

The chromosomal constitution of Dictyostelium discoideum was first described by Wilson (1952, 1953). He prepared aceto-orcein stained squashes of amoebae taken from migrating pseudoplasmodia and observed mitotic figures with haploid complements of 7 chromosomes. Bonner \& Frascella (1952) reported a chromosome number of 4 with three extra arms but this difference was subsequently resolved and Wilson's conclusion has since been amply confirmed by Wilson \& Ross (1957) and by R. R. Sussman (1961). The latter used a fixing and staining procedure which permitted immediate diagnosis of ploidy in exponentially growing organisms as well as those that had divided during the fruiting process. In 1957, Wilson \& Ross observed the presence of diploid cells in D. discoideum cultures. Ross (1959) isolated strains which, although not subjected to clonal analysis, provided evidence that the diploid state is heritable. On several occasions he obtained haploid stocks from the diploid and vice versa, but was unable to define environmental conditions which could reproducibly induce or select the ploidal conversions. Sussman, Sussman \& Ennis (1960) were able to isolate metastable strains containing appreciable numbers of both haploid and diploid cells. They arose as clones from I-cells, a minor component of the haploid myxamoebal population distinguishable by size, flatness, motility, granulation, etc. The capacity of these metastable strains to yield a ploidal mixture was found to be stably inherited through numerous subcultures and clonal re-isolations. The present communication describes the properties of the three ploidal varieties, in particular, the differences in the size distribution of spores and myxamoebae, I-cell frequencies, aggregative performances, and the stability of ploidal inheritance. It also specifies the environmental conditions which permit the isolation of one strain from another. 


\section{METHODS}

Organisms and cultivation. Dictyostelium discoideum strain NC-4 is a stable haploid stock used in our laboratory for many years. Its genetic homogeneity was maintained by routine clonal re-isolations. Strain RA is a stable diploid stock which appeared in the laboratory of Dr K. B. Raper and which he kindly sent to us for study. The remaining strains were derived from these. Cultivation was accomplished on SM agar in association with Aerobacter aerogenes (Sussman, 1951).

Size distributions. Myxamoebae were harvested at the beginning of the stationary phase after incubation for $40-44 \mathrm{hr}$. at $22^{\circ}$, washed three times by centrifugation, suspended in water and dispensed in $0.01 \mathrm{ml}$. samples on washed agar at a density of 150-200 cells $/ \mathrm{mm}^{2}$. After incubation for 4-5 hr. at $22^{\circ}$, the amoebae were either measured directly with an ocular micrometer, or photomicrographs were taken using polaroid transparency film. These were projected on a screen and the major and minor cell diameters measured directly from the projected images and calibrated with a photomicrograph of an ocular micrometer. Both methods yielded the same size distributions. Care had to be taken to standardize the age of the organisms and conditions of cultivation and pretreatment in order to obtain reproducible data. Previous work (Bonner, 1960) had shown that amoebal size was significantly affected by these factors. Spores to be measured were taken from mass or clonal plates after incubation for 4-6 days and were measured with an ocular micrometer in wet mounts under an oil-immersion objective.

Chromosome stains. The procedure used is reported elsewhere (R. R. Sussman, 1961). The amoebae were fixed in Carnoy solution, brought to water through an ethanol series and were hydrolysed in $\mathrm{N}-\mathrm{HCl}$ for 9-10 min. at $60^{\circ}$. A quick wash with $45 \%(\mathrm{w} / \mathrm{v})$ acetic acid was followed by staining in aceto-orcein. Squashed wet mounts could be examined immediately under phase contrast or sealed and retained for later examination.

Aggregative performance. This was determined as described by Sussman \& Sussman $(\mathbf{1 9 6 1} b)$. Myxamoebae were harvested from growth plates, washed three times by centrifugation, suspended in chilled water and counted in a Levy chamber. Appropriate dilutions were dispensed on washed agar in drop volumes ranging between 0.01 and $0.2 \mathrm{ml}$. in order to deliver a constant number of myxamoebae over a range of population densities. After incubation for 18-24 hr., counts were made of the number of aggregative centres.

\section{RESULTS}

\section{Chromosome figures of stable haploid and diploid strains}

Myxamoebae were harvested from growth in the exponential phase, suspended in salt solution and dispensed on a slide. The amoebae adhered to the glass and could be examined at intervals microscopically. During the following $3 \mathrm{hr}$., an appreciable proportion of the amoebae rounded up in a fashion characteristic of mitosis. They were immediately fixed in Carnoy solution and stained. When the fixation was timed correctly, considerable numbers of metaphase figures could be observed in the squashed stained preparations, thus permitting precise chromosome counts. In all, several hundred metaphase figures were scored. No haploids were encountered in strain RA, no diploids in strain NC-4. 


\section{Size distributions of strains $N C-4$ and $R A$}

Preliminary data of Ross (1959) indicated a significant difference between the mean sizes of haploid and diploid myxamoebae. The size distributions of RA and NC-4 myxamoebae (see Fig. 1), confirmed this conclusion.

Figure 2 summarizes the size distributions of RA and NC-4 spores, and shows that spore size was also affected by ploidy. The major diameter in micrometer divisions ( 1 div. $=0 \cdot 83 \mu$ ), was used as the criterion of size since the minor diameters
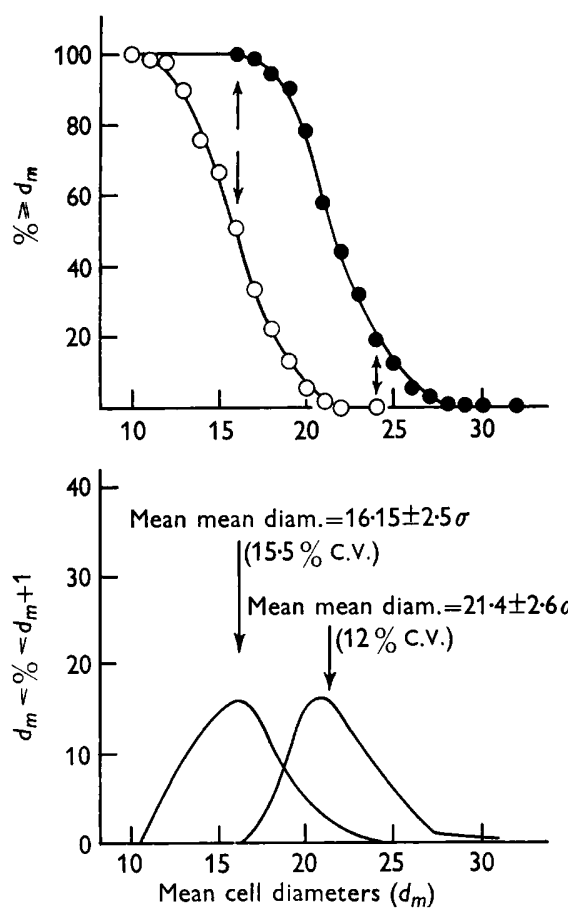

Fig. 1
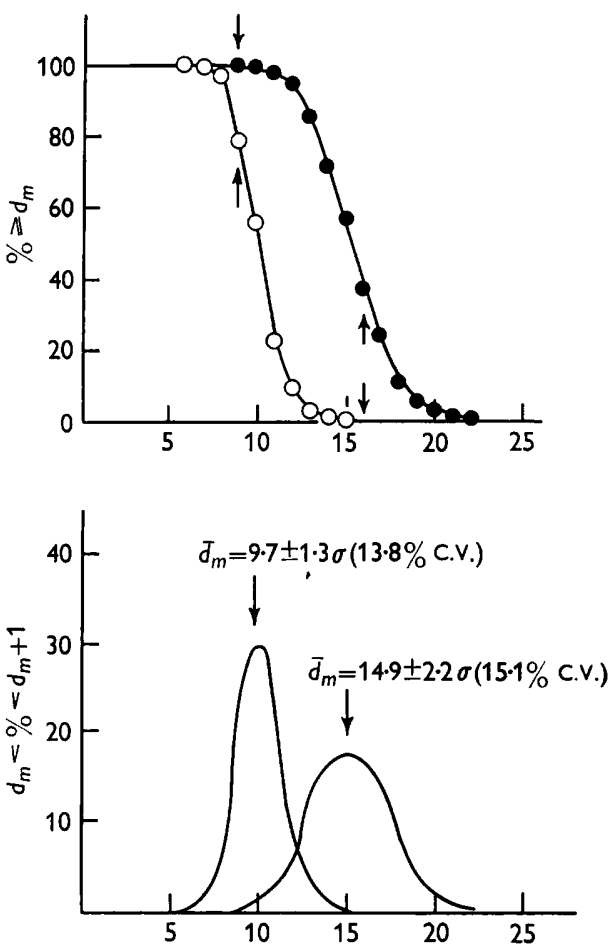

Major diameters $\left(d_{m}\right)$ in micrometer divisions

Fig. 2

Fig. 1. Size distributions of NC-4 and RA myxamoebae. Upper curves show the percentage amoebae whose mean diameters equalled or exceeded the values given in the abscissa. Lower curves are histograms derived from 1-A. They show the percentage amoebae falling within unit increments in the mean diameters given in the abscissa. The arrows delineate the zones of exclusivity, $49 \%$ of the NC-4 amoebae were smaller than the smallest RA; $19 \%$ of the RA amoebae were larger than the largest NC-4. O, NC-4 (haploid) 262 amoebae; O, RA (diploid) 326 amoebae.

Fig. 2. Size distributions of NC-4 and RA spores. The method of plotting is the same as used in Fig. 1 except that the units of the abscissae are major diameters in micrometer divisions (1 div. $=0 \cdot 83 \mu$ ). $\bigcirc, \mathrm{NC}-4$ (haploid), 583 spores;, RA (diploid), 1049 spores.

differed only slightly (5.4 against $5 \cdot 05$ micrometer divisions). The zone of exclusivity at the low end of the scale included $23 \%$ of the NC-4 spores and at the high end, $38 \%$ of the RA spores. Considering the spores as perfect cylinders, the respective mean volumes were 201 and $115 \mu^{3}$, yielding a volume ratio of about 1.75. The 
photomicrographs of RA and NC-4 spores in Pl. 1, figs. 1 and 2, show differences in shape as well as size. The haploid spores are regular cylinders, whereas among the diploid spores many are banana- or boomerang-shaped and otherwise irregular in outline.

The data for strain RA shown in Fig. 2 were obtained in five separate experiments which yielded a grand mean major diameter of $14.9 \mathrm{div}$. The coefficient of variation was $3.7 \%$, indicating that examination of as few as 100-200 spores can provide a reliable estimate of mean cell size.

\section{The frequency of I-cells}

When myxamoebae, taken from log phase or early stationary phase cultures of strain NC-4, are inspected microscopically an occasional amoeba stands out in dramatic contrast to its neighbours by virtue of its large size, flatness, granulation and motility (Ennis \& Sussman, 1958b). Frequently it contains one or more engulfed amoebae. For convenience, these exceptional myxamoebae were designated 'I-cells', and the remainder of the population ' $R$-cells'. It is not clear whether they represent a truly dichotomous type or are merely the extreme of a continuum of cell sizes and associated properties. Under the conditions used for measurement, a mean diameter of $28 \mu$ was set arbitrarily as the lower limit for I-cells, and they have been seen to range up to $50 \mu$ (in contrast to the mean of mean diameters $=16 \mu$ for strain NC-4 as a whole). It is possible to show that the I-cells possess a capacity to initiate centres of aggregation greatly superior to that of the rest of the population, and that during their growth, they frequently undergo multiple fissions to yield three or four daughters. As shown below, they can yield clones which contain substantial numbers of both diploid and haploid myxamoeba.

In strain NC-4, the proportion of I-cells was found to be about 1 in 2000 under specified conditions of cultivation (Ennis \& Sussman, 1958b). In strain RA under comparable growth and sizing conditions, about $1 \%$ of the population had a mean amoebal diameter greater than or equal to $28 \mu$ (see Fig. 1) and possessed a degree of flatness, granulation and explosive motility equivalent to the myxamoebae classified as I-cells within the haploid population. However, in strain RA the size distribution in this range was such that a diploid population, unlike its haploid counterpart, did not show a dichotomous appearance upon microscopical inspection.

\section{Ploidal stability of strains $\boldsymbol{R A}$ and $\mathrm{NC}-\mathbf{4}$}

As mentioned above, examination of mitotic figures led to the conclusion that the frequencies of diploid myxamoebae in strain NC-4 and of haploid myxamoebae in strain RA were probably of the order of one in several hundred myxamoebae or less. This point was investigated further. Both strains were plated clonally (Sussman, 1951) and wet mounts of spore samples, each from a single clone, examined microscopically. Major diameters of 20 randomly chosen spores were recorded for some of the clones, to obtain a rough measure of mean size. Thus, among 23 clones of RA, the mean of mean major diameters was $\mathbf{1 5 . 3}$ div., in good agreement with the value given in Fig. 2. In addition, a few hundred spores from each clone were scanned to detect the presence of abnormally small spores (major diameter less than 8 div.) in strain RA or abnormally large spores (major diameter greater than or equal to 
18 div.) in strain NC-4. In practice the difference between stable haploid and diploid spore populations was clear enough to be readily apparent by simple inspection under the microscope and the spore measurements served merely to confirm the diagnoses numerically. Of $373 \mathrm{NC}-4$ clones examined in this fashion, none was diploid, i.e. they corresponded closely to the size distribution shown in Fig. 2 for strain NC-4 with respect to mean spore size and incidence of abnormally large spores, and with the spores shown in Pl. 1 with respect to shape. Of 4110 strain RA clones, none was haploid, by the same criteria. In neither case were distributions intermediate between the two extremes observed.

During these and similar examinations, at least $10^{4}$ spores of each strain were scanned without finding a single spore with major diameter less than 8 div. in strain RA or one greater than or equal to 18 div. in strain NC-4. Figure 2 indicates that about $10 \%$ of the NC-4 spores had a major diameter less than 8 div. and about $10 \%$ of the RA spores greater than or equal to $18 \mathrm{div}$. Therefore, it can be argued that since the incidence of small spores in strain RA and large spores in strain NC-4 was less than one in $\mathbf{1 0}^{\mathbf{4}}$, the total incidence of haploid cells in $\mathbf{R A}$ and of diploid cells in $\mathrm{NC}-4$ must have been of the order of one in a thousand or less.

The validity of either method of estimation described above rests on the assumption that a ploidal change would necessarily have been accompanied by a change in spore size; this assumption was checked without contradiction many times during the isolation of haploid and diploid strains against the actual chromosome complements. The inspection of $\mathrm{RA}$ and NC-4 clones with respect to spore size distributions thus extended the results obtained by direct chromosomal counts. The incidence in strain RA of myxamoebae capable of yielding haploid clones, and in strain NC-4 of myxamoebae capable of yielding diploid clones, must be less than one in several hundred. Also, the absence of abnormally large spores in strain NC-4 and of abnormally small spores in strain RA suggests that the incidence of ploidal variants may be of the order of one in a thousand or less.

\section{Isolation of a stable diploid strain from $\mathrm{NC}-4$}

In light of the discovery (Ross, 1959) of stable diploid and metastable strains of Dictyostelium discoideum, stocks carried in a number of laboratories were reexamined for ploidal composition (Dr I. K. Ross; private communication). The prevalence of diploid stocks found by Dr Ross in some laboratories, regardless of the original source of the strains, suggested that the diplophase might be selected or induced by conditions of cultivation. We examined this point and this indeed turned out to be the case. Spores from a typical culture of strain NC-4 were inoculated at the centre of a cross streak of bacteria on SM agar after the method of Raper (1951). The myxamoebae grew out along the arms of the streak during the next 3-4 days. The resulting fruits revealed a spore-size distribution characteristic of the stable haplophase. Serial subculture with spores as inoculum did not alter this condition. When, however, the inoculum consisted of a mass of spores, stalks, partially complete fruits and unaggregated myxamoebae swept up from an agar surface and deposited at the centre of a cross-streak, the ensuing fruits often (not always) contained a small number (0.1-1.0\%) of abnormally large spores (major diameter greater than or equal to $\mathbf{1 7}$ div.), i.e. within the zone of exclusivity of stable diploids. The proportion was further enriched by a second serial cultivation 
as described above. These spores were plated clonally and a majority of the clones displayed spore size distributions characteristic of the stable diplophase. The remaining clones were typically haploid. No intermediate distributions were detected. One of the presumptive diploid clones was retained and designated NC-4 dip-1; chromosome counts confirmed that this was indeed a stable diploid. Figure 3 demonstrates that the spore size distribution of this stock is identical to RA within the limits of experimental and sampling errors.
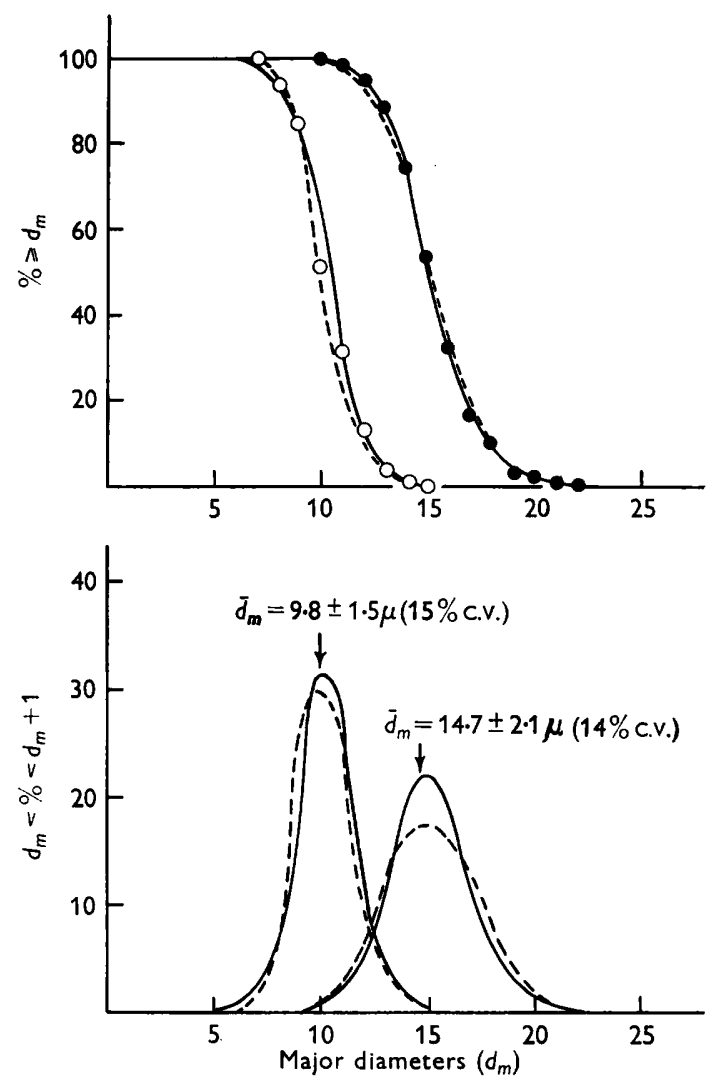

Fig. 3. Size distributions of spores from NC-4 dip-1 and NC-4 hap-1. For comparison, the distributions of NC-4 and RA spores taken from Fig. 2 are given as dotted lines. $\bigcirc$, NC-4 hap-1, 100 spores; 9 , NC-4 dip-1, 109 spores.

\section{Selection of stable haploid clones from strains $R A$ and NC-4 dip-1 during sustained exponential growth}

An inoculum of 1-2 $\times 10^{5} \mathrm{RA}$ my xamoebae spread with Aerobacter aerogenes on an SM agar plates grows exponentially with a generation time of $\mathbf{3 . 9} \mathrm{hr}$., attains the stationary phase after ten to twelve generations, and then aggregates and fruits. Strains RA and NC-4 dip-1 were both cultivated in this manner but were subcultured before the onset of aggregation once again, at an initial density of $1-2 \times 10^{5}$ myxamoebae/plate. The sampled plates were incubated further in order that the spores therefrom could be inspected microscopically. Four serial subcultures were 
accomplished in this fashion. Table 1 shows the incidence of abnormally small spores in each subculture.

After the 4th subculture the spores were plated clonally. Of 19 clones examined, 14. were typical RA clones with respect to mean spore size and absence of abnormally small spores, while 5 were typically haploid in these respects. Three of the latter clones were retained for study. Chromosome counts yielded haploid figures only and examination of spores from 20 to 40 clones subsequently derived from each stock indicated that the haplophase was stably inherited. Stable haploid strains were also derived from the diploid stock NC-4 dip-1 (see the preceding section) recognized first on the basis of spore size and later confirmed by chromosome counts. The spore size distribution of one of these, designated NC-4 hap-1 (Fig. 3) was identical with the original parent (the haploid NC-4) from which the diploid NC-4 dip-1 had itself been derived.

Table 1. Incidence of small spores in Dictyostelium discoideum strain $\boldsymbol{R A}$ after sustained logarithmic growth

The symbol $d_{m}=$ major diameter in micrometer divisions.

\begin{tabular}{ccccccc} 
Subculture & $\begin{array}{c}\text { Generations } \\
\text { (cumulative) }\end{array}$ & $\overbrace{\text { Proportion }}^{\text {Spores with } d_{m} \leqslant 9}$ & & \multicolumn{2}{c}{ Spores with $d_{m} \leqslant 8$} \\
0 & 0 & & & Proportion & $\%$ \\
2 & 22 & $5 / 1049$ & $0 \cdot 3$ & & $0 / 1049$ & 0 \\
3 & 33 & $10 / 430$ & $1 \cdot 2$ & & $3 / 421$ & $0 \cdot 7$ \\
4 & 44 & $16 / 234$ & $6 \cdot 3$ & & $6 / 430$ & $1 \cdot 4$ \\
& & & $6 \cdot 8$ & & $7 / 234$ & $3 \cdot 0$
\end{tabular}

\section{Aggregative performances of diploid and haploid strains}

Myxamoebae, washed free of bacteria and dispensed on washed agar, remain constant in number and can produce typical radiate aggregative patterns and, ultimately, normal fruits. Under these conditions, the number of centres of aggregation is a function of the number of myxamoebae present and their population density. The 'aggregative performance' of a particular strain was defined by Sussman \& Sussman $(\mathbf{1 9 6 1} b)$ as the specific relation between the number of centres and the parameters of cell number and population density. When carried out under comparable experimental conditions, this relation can serve as a measure against which can be assessed the effect of genetic and physiological variation, of added metabolites, enzyme poisons, etc., upon the morphogenetic sequence.

Table 2 compares numerically the aggregative performances of strains RA, NC-4, NC-4 dip-1 and NC-4 hap-1. The general shapes of the curves are published elsewhere (Sussman \& Sussman, 1961 b). Significant differences between strains NC-4 dip-1 and NC-4 hap-1 are apparent in respect to the optimal population density for centre formation (100 versus 200 myxamoebae $/ \mathrm{mm}^{2}$ ) and the ratio between the number of centres formed at the optimal population densities and the number of myxamoebae present $(1: 400$ versus $1: 2000)$. In contrast, the performance of NC-4 hap-I was identical with that of the original parental stock, NC-4 (optimal population density $=200$ myxamoebae $\left./ \mathrm{mm}^{2} ; R=1: 2200\right)$. Thus the shift from the stable haplophase (NC-4) to the stable diplophase (NC-4 dip-1) was accompanied by a considerable change in aggregative performance; and the subsequent reversion of NC-4 dip-1 to the haplophase (NC-4 hap-1) returned this performance to its 
original condition. Because myxamoebal size has already been shown to be associated with differing morphogenetic capacities (Ennis \& Sussman, 1958b) it may be that the myxamoebal size distribution is the determining factor here. It should be noted that the aggregative performance of strain RA was also found to be different from that of strain NC-4. However, the pedigree of RA is uncertain and since it is known that genetic alterations aside from ploidy can affect aggregative capacity (Sussman \& Sussman, 1961 b), the above difference cannot be ascribed uniquely to the change in ploidy.

\section{Table 2. Aggregative performances of haploid and diploid strains of Dictyostelium discoideum}

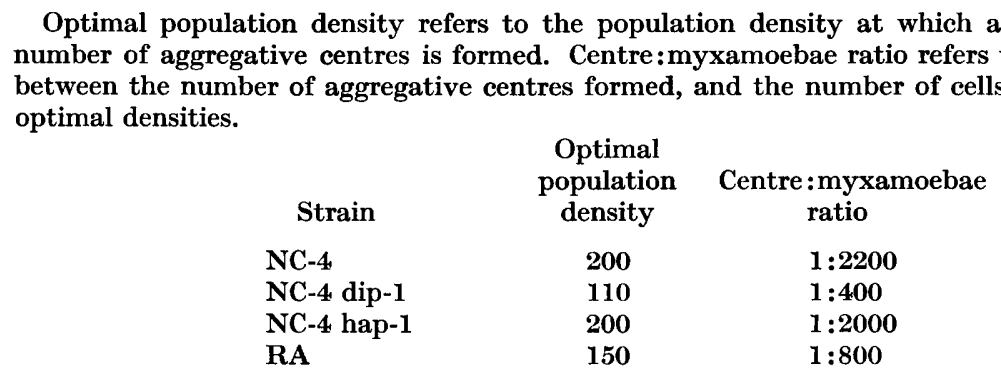

\section{Metastable strains}

In previous work (Sussman et al. 1960), NC-4 myxamoebae classified as I-cells were isolated by micro-manipulation and incubated on agar with Aerobacter aerogenes. Examination of the resulting clones revealed that about $90 \%$ were indistinguishable from those derived either from $\mathbf{R}$-cell isolates or from the carried stock culture on the bases of size distributions of spores and myxamoebae, frequency of I-cells, aggregative performance and ploidy. (All countable metaphase figures were haploid.) In contrast, about $10 \%$ of the I-cells yielded clones which differed from the norm in all the respects noted above and which inherited the anomalies in stable fashion. These anomalous stocks, hereafter termed 'metastable', have now been further studied; current information about them is summarized below.

Ploidal composition. Three metastable strains were examined cytologically; Table 3 shows the data. All displayed a mixture of haploid and diploid metaphase figures, but in very different proportions. The amplitude of transient variations in the proportion of haploid and diploid myxamoebae during the culture cycle is not known with precision but is probably considerable. Nevertheless, the strains tended to be sorted into two classes : those with a preponderance of haploid myxamoebae and those with a preponderance of diploid. These proportions tended to be maintained during serial subculture and clonal re-isolation.

Size distributions. Figure 4 shows the spore distribution encountered in strain I-2A, compared with the corresponding curves for $\mathrm{NC}-4$ (the haploid stock from which I-2A was derived) and NC-4 dip-1 (a diploid stock of similar origin). In rough agreement with the cytological findings, the size distribution is seen to lie between the two extremes of ploidal stability. Figure 5 summarizes the spore-size distribution of three other metastable strains. I-47 had been isolated from a mutant 
of Dictyostelium discoideum, designated $\mathrm{Br}-1$, which produces the yellow pigment characteristic of the wild type in its fruits, but also forms a brown pigment which stains deeply both the fruits and the underlying agar. I-262 and W-7DH came from

Table 3. Ploidal composition of metastable strains of Dictyostelium discoideum

$\begin{array}{lccc}\text { Metaphase } & & \\ \text { figures } & & \text { No. haploid } & \text { No. diploid } \\ \text { scored } & 40 & \mathbf{3} & \mathbf{3 7} \\ \text { I-2A } & \mathbf{1 1 2} & \mathbf{1 0 0} & \mathbf{1 2} \\ \text { I-47 } & \mathbf{4 4} & \mathbf{1} & \mathbf{4 3}\end{array}$
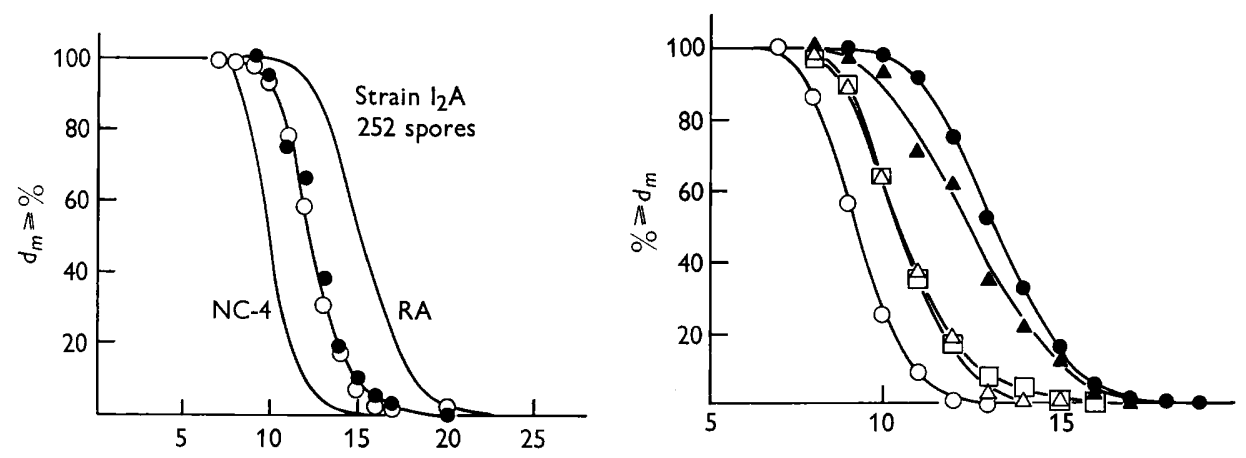

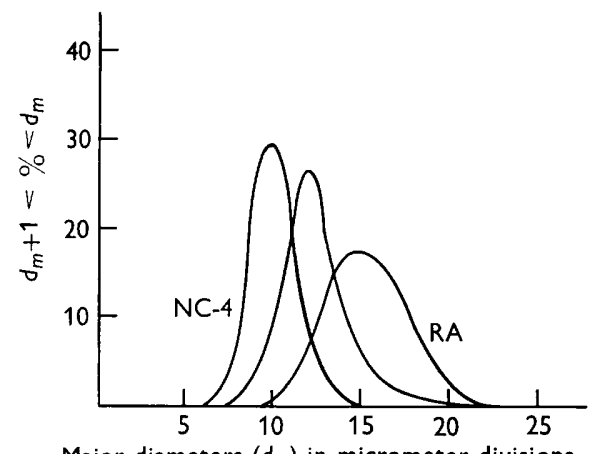

Major diameters $\left(d_{m}\right)$ in micrometer divisions

Fig. 4

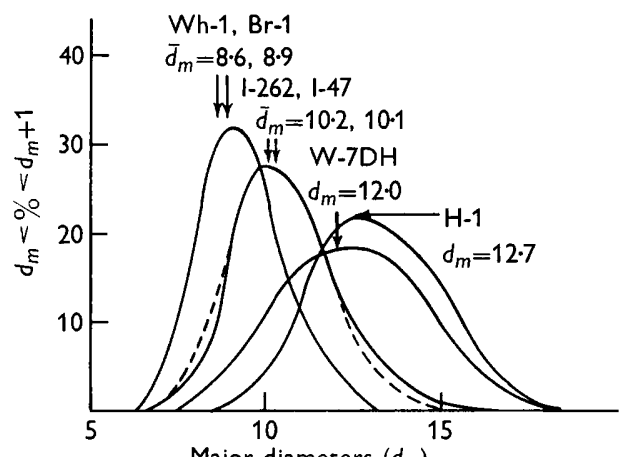

Major diameters $\left(d_{m}\right)$

Fig. 5

Fig. 4. Size distribution of the spores of I-2A, a metastable strain. The open and closed circles represent data from separate determinations made 11 months apart. The distributions of NC-4 and RA spores, taken from Fig. 2, are given for comparison.

Fig. 5. Size distributions of spores from the following strains of $D$. discoideum : $\mathrm{Br}-1$, a yellow-brown mutant, and Wh-1, a white mutant, both stable haploids; I-47, a metastable strain derived from $\mathrm{Br}-1 ; \mathrm{I}-262$ and $\mathrm{W}-7 \mathrm{DH}$, metastable strains derived from Wh-1; H-1, a diploid strain isolated from a mixed culture of I-47 and I-262. $O$, Wh-1, Br-1, 186 spores;, H-1, 721 spores; $\triangle$, I-47, 253 spores; $\Delta, W-7 D H, 150$ spores; $\square$, I-262, 135 spores.

a white mutant Wh-1 (Sussman \& Sussman, 1953). Strain H-1 is a stable diploid; it was derived from a mixed culture of I-477 and I-262. Although most H-I clones were diploid and had the wild-type pigmentation (yellow), haploid segregant clones appeared at low frequency (about $1 / 1000$ ) which had either of the parental pheno- 
types (white or yellow-brown) or either of the recombinant phenotypes (yellow, or white-brown). These phenotypes are described elsewhere (Sussman, 1961). Both parental stocks, $\mathrm{Br}-1$ and $\mathrm{Wh}-1$, formed spores slightly but significantly smaller than wild-type haploid NC-4. The derived H-1 spores were in turn correspondingly smaller than those of NC-4 dip-1, for example. The metastable spores were distributed intermediately between the two ploidal extremes of this series of stocks, in rough correspondence with the proportions of haploid and diploid cells observed cytologically.

Frequency of I-cells. Casual microscopic inspection of myxamoebae from metastable stocks revealed a degree of morphological heterogeneity more marked than in stable haploid populations (Pl. 1, fig. 3). This appeared to be due in part to a much greater frequency of myxamoebae classifiable as I-cells (as much as $1 \%$ of the I-2A population) which appeared in dramatic contrast to their smaller neighbours and in part to the generally greater size of the metastable I-cells as compared with those in the haploid NC-4 stock.

Aggregative performance. Data published elsewhere (Sussman \& Sussman, $1961 b$ ) showed that the performances of strains I-2A and I-47 were significantly different from that of the parental NC-4. Both metastable stocks showed an optimal population density less than 100 myxamoebae $/ \mathrm{mm}^{2}$ (as compared with 200 for NC-4). At optimal density the centre:myxamoebae ratios were $1: 530$ for $\mathrm{I}-2 \mathrm{~A}$ and $1: 830$ for I-47 (as compared with $1: 2200$ for NC-4).

Ploidal stability. Clones produced by three metastable stocks (I-2A, I-47, and $\mathrm{W}-7 \mathrm{DH})$ were examined for spore size. Of particular interest in this respect was I-47. Cytological examination of I-47 indicated a proportion of about 1 diploid myxamoeba to 10 haploids in growing populations. Yet when representative samples were plated out, every clone of over a hundred examined contained spores large enough ( $d_{m}=17$ divisions) to fall within the zone of exclusivity for diploids. No stable haploid clones were detected. It therefore appears that haploid myxamoebae in I-47 differed from haploid myxamoebae in NC-4 in terms of the rate at which they gave rise to diploid progeny. The term metastable seems to be an appropriate designation for such strains. Metastable stocks have been found to yield both stable haploid and stable diploid derivatives and by the same methods of cultivation as were described above. Stock I-2A was cultivated continuously in the log phase for twenty-four generations and a sample plated on SM agar with Aerobacter aerogenes. Six percent of the clones were diagnosed as stable haploids by spore size and confirmed by cytological examination. None of the zero-time clones was haploid. Stock I-47 was cultivated from an inoculum of spores, stalks, immature fruits and unaggregated cells. Stable diploid derivatives were isolated after a single subculture in this manner (about ten generations). During routine laboratory passage the stock W-7DH was discovered to have undergone a considerable heritable alteration in spore size distribution toward the haploid extreme. Cytological observation revealed only 3 diploid myxamoebae out of 12 countable metaphase figures. At this time myxamoebae classified as I-cells were isolated from the culture by micromanipulation. A low proportion of these yielded clones which had regained a preponderantly diploid state as indicated by spore size and cytological examination. Similarly, isolation of I-cells from I-47 yielded a low proportion of clones in which the diplophase predominated and the alteration was heritable. 


\section{DISCUSSION}

Previous cytological examination of Dictyostelium discoideum had revealed the existence of haploid and diploid myxamoebae and had indicated that the ploidal condition might be stably inherited. The present work has confirmed these findings and supports the inference that ploidal constitution is a clonally inherited property in this species. It has also shown the existence of strains which display a wide range of ploidal compositions. For example, in strains NC-4, BR-1 and Wh-1, all scorable metaphase figures out of hundreds examined were haploid. Subsidiary evidence indicated that diploid cells might be present at a frequency of about $\mathbf{1}$ in $\mathbf{1 0 0 0}$ or less in these strains. In strains RA, NC-4 dip-1 and H-1, all scorable metaphase figures were diploid and subsidiary evidence indicated that haploid myxamoebae might be present at a frequency of about 1 in 1000 or even less. In strains I-2A and W-7DH, both haploid and diploid figures were encountered but the diploid predominated (about 90-98\%) while in strain I-47, the haploid predominated (about $90 \%$ ). Subsidiary evidence suggests that in these strains the presence of both haploid and diploid cells in appreciable proportions is due to high rates of ploidal variation.

The three groups have been termed respectively, 'stable haploid', 'stable diploid', and 'metastable' strains and their ploidal properties have been found to be heritable and clonally distributed. It may be that a more detailed analysis of these and other stocks will demonstrate the existence of a continuous spectrum of ploidal compositions among isolates of Dictyostelium discoideum, in which case any categorization would be arbitrary. Nevertheless, the above nomenclature is offered as a convenient one and would seem to be justified at present since the relative proportions of haploid and diploid cells in the three groups differ by almost two orders of magnitude and they appear to retain these differences during serial passage under identical conditions of cultivation.

A correlation between cell size and ploidy has been reported for a wide variety of plant, animal, and microbial cells including at least two groups of Fungi (Sansome, 1949; Roman \& Sands, 1953). It is therefore not unexpected to find this correlation operative in Dictyostelium discoideum. It should be emphasized, however, that size can be affected genetically without changes in chromosome number and the difference among the haploid stocks used in this study is a case in point. A previously encountered example is the mutant Agg-91, a dwarf aggregateless derivative of NC-4 (Ennis \& Sussman, 1958a). In addition, the size of amoebae is to some extent governed by their physiological condition (Bonner, 1960). It is important, therefore, that comparisons between strains be made with these parameters in mind.

It is now possible to stipulate experimental procedures by which each of the three types of ploidal strains can be isolated, one from another. But it still remains unclear how the ploidal variants are thereby induced or selected. Furthermore, the genetic basis for inheritance of a particular range of ploidal composition is completely unknown and is an intriguing problem. Finally, neither the act of diploidization nor of haploidization is understood. One datum of significance is the existence of stable diploids like strain $\mathrm{H}$-1, isolated from a mixed culture of two mutants and apparently heterozygous for both of the mutant markers which segregate during 
haploidization (Sussman 1961). This indicates that at least some diploids can arise as the result of fusion and karyogamy between independent amoebae, as has been suggested by Wilson \& Ross (1957). The ecological consequences of ploidal variation for Dictyostelium discoideum cannot be definitively estimated at present. The original species description of $\boldsymbol{D}$. discoideum (Raper, 1935) suggests that the first isolate was a stable haploid. Stocks collected subsequently appear to have been either stable haploids or possibly metastable but certainly containing few diploids (Dr I. K. Ross, private communication). The only stable or largely diploid metastable strains now extant were derived in the laboratory from pre-existing haploids. However, only a relatively narrow range of natural habitats has thus far been investigated, by restricted methods of isolation, and only during certain seasons of the year (Raper, 1951). The realization that this species can exist in the diplophase may now prompt an examination of its ecological significance.

One final consideration is noteworthy. The data have demonstrated that ploidal variation is accompanied by a gamut of changes in morphology and morphogenetic behaviour. Because ploidal variants appear at appreciable frequencies in stock cultures, they constitute an additional danger to experimental reproducibility over and above the incidence of ordinary genetic variants.

\section{Supported by Grants G-12900 (NSF) and C-4057 (NIH).}

\section{REFERENCES}

Bonner, J. T. (1960). Developing cell systems and their control. 18th Groreth Symposium, Soc. for Study of Development and Grozth, p. 3. Minneapolis, Min., U.S.A.: Ronald Press.

Bonner, J. T. \& Frascella, E. (1952). Mitotic activity in relation to differentiation in Dictyostelium. J. exp. Zool. 121, 561.

Ennis, H. L. \& Sussman, M. (1958a). Synergistic morphogenesis by mixtures of $D$. discoideum wild type and aggregateless mutants. J. gen. Microbiol. 18, 433.

Ennis, H. L. \& Sussman, M. (1958b). The initiator cell for slime mold aggregation. Proc. nat. Acad. Sci., Wash. 4, 401.

RAPER, K. B. (1935). D. discoideum, a new species of slime mold. J. agric. Res. 50, 135.

RAPER, K. B. (1951). Isolation, cultivation and conservation of simple slime molds. Quart. Rev. Biol. 26, 169.

Roman, H. K. \& Sands, S. M. (1953). Heterogeneity of clones of saccharomyces derived from diploid ascospores. Proc. nat. Acad. Sci., Wash. 39, 171.

Ross, I. K. (1959). Diploid strains of D. discoideum. Amer. J. Bot. 47, 54.

Sansome, E. (1949). Diploid strains of Aspergillus. Trans. Brit. mycol. Soc. 32, 305.

Sussman, M. (1951). Origin of cellular heterogeneity in Dictyosteliaceae. J. exp. Zool. $118,407$.

SusSman, M. (1961). Genetic and morphogenetic consequences of an act of cellular differentiation in the slime molds. Purdue Univ. Symp. on Growth. Brooklyn, N.Y., U.S.A.: Basic Books Publ.

Sussman, R. R. (1961). A method of staining the chromosomes of D. discoideum. Exp. Cell Res. 24, 154.

Sussman, M. \& Sussman, R. R. (1953). Cellular differentiation in Dictyosteliaceae. Ann. N.Y. Acad. Sci. 56, 949.

Sussman, M. \& Sussman, R. R. (1961 $a$ ). Haploidization of diploid strains of D. discoideum. Bact. Proc. p. 100.

Sussman, M. \& Sussman, R. R. (1961b). Aggregative performance. Exp. Cell Res. Suppl. 8, 91.

Sussman, M., Sussman, R. R. \& Ennis, H. L. (1960). The appearance and inheritance of the I-cell phenotype in D. discoideum. Develop. Biol. 2, 367. 


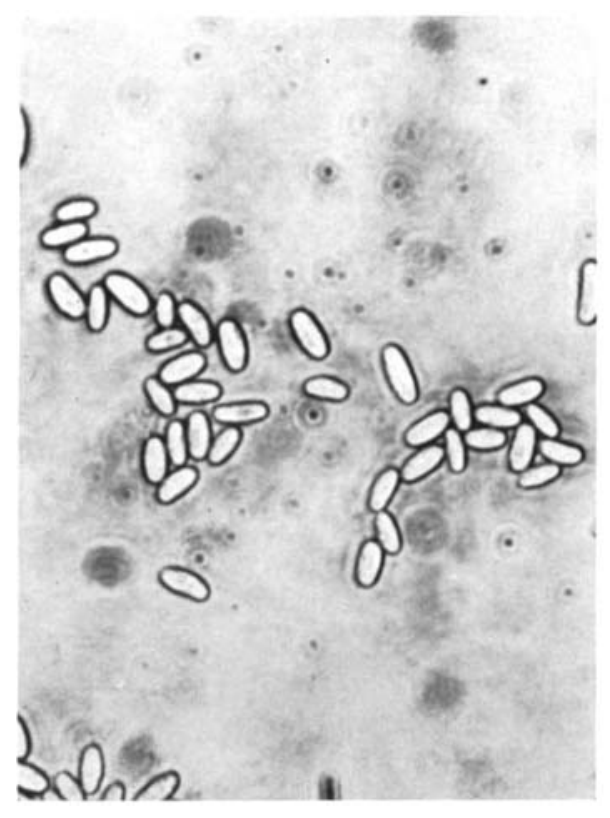

Fig. 1

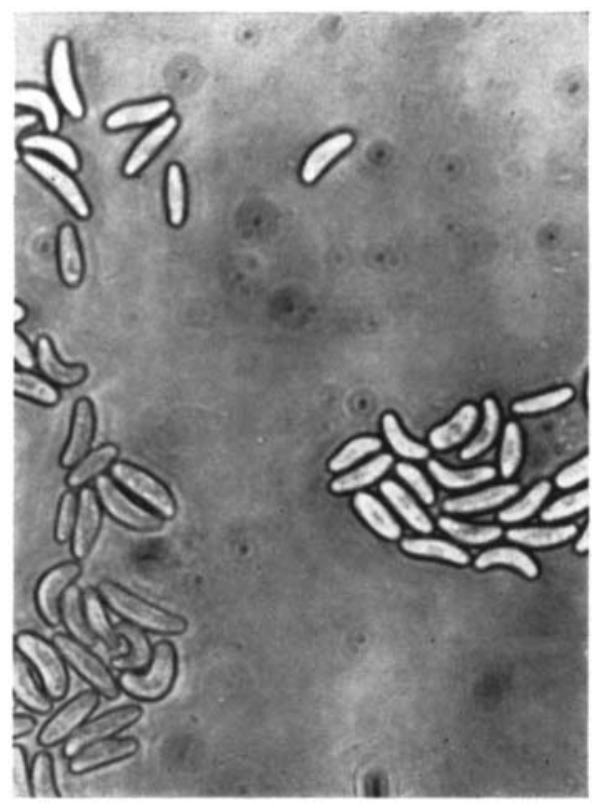

Fig. 2

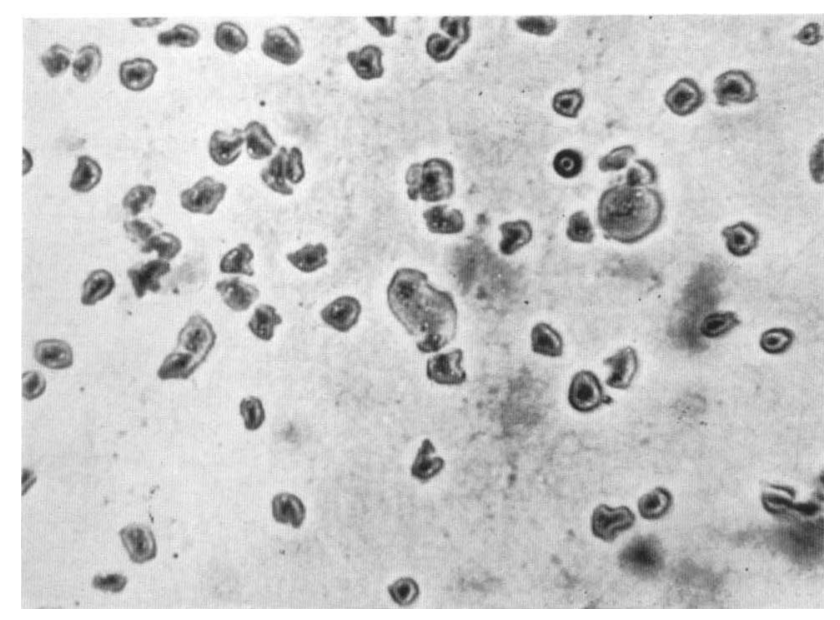

Fig. 3 
Wilson, C. M. (1952). Sexuality in the Acrasiales. Proc. nat. Acad. Sci., Wash. 38, 659. WiLson, C. M. (1953). Cytological study of the life cycle of Dictyostelium. Amer. J. Bot. 40, 714.

Wilson, C. M. \& Ross, I. K. (1957). Further cytological studies in the Acrasiales. Amer. J. Bot. 44, 345.

\section{EXPLANATION OF PLATE}

Plate 1

Figs. 1 and 2. Photomicrographs of NC-4 spores (left) and RA (right) to show the differences in shape. Both are at $\times 1000$.

Fig. 3. A photomicrograph of W-7DH myxamoebae. The mean diameter of these cells was $c .20 \mu$, but the two largest myxamoebae had mean cell diameters of 44 and $38 \mu$, respectively. $\times 240$. 